\title{
3-D hydrodynamical simulations of SNR-clouds and SNR-SNR interactions in Starburst environments
}

\author{
Claudio Melioli ${ }^{1}$, E. M. de Gouveia Dal Pino ${ }^{1}$ and A. Raga ${ }^{2}$ \\ ${ }^{1}$ IAG-USP, Cidade Universitaria, R. do Matao, 1226 05508-090 São Paulo, SP, Brazil \\ email: cmelioli@astro.iag.usp.br, dalpino@astro.iag.usp.br \\ ${ }^{2}$ Instituto de Ciencias Nucleares, UNAM, Apdo. 70-543, 04510 D. F., Mexico
}

\begin{abstract}
We study the global effects of the clouds-SN shock wave interactions on the mixing of the gas in Starburst galaxies.
\end{abstract}

Most galaxies present supernova (SN) shock fronts interacting with a cloudy interstellar medium (ISM). These interactions can occur either at small scales, between a single supernova remnant (SNR) and a compact cloud or globule, or at large scales, between a giant shell of a superbubble and a molecular cloud. Particularly, in this work we are interested to study the by-products of SNR-clouds and SNR-SNR interactions in a starburst (SB) ambient. Due to the high SN rate in this environment, a cloud may be shocked more than once by SNRs. These interactions can have an important role in the recycling of matter from the clouds to the ISM and vice-versa (see, e.g., Melioli \& de Gouveia Dal Pino 2004a, 2004b). This study is also relevant to understand the evolution of the ISM density and the structure of the clouds embedded in it.

In the presente work, we have focused our attention on the global effects of the interactions between clouds and SNR shock fronts in the ISM, and performed three-dimensional (3D) numerical simulations employing a modified version of the Yguazú-a adaptive grid code originally developed by Raga, Navarro-Gonzalez \& Villagran-Muniz (2000; see also Raga et al. 2002, Masciadri et al. 2002, Gonzalez et al. 2004). This code integrates the hydrodynamic equations explicitly accounting for the radiative cooling together with a set of continuity equations for several atomic/ionic species employing the flux-vector splitting algorithm of Van Leer. The following species have been considered: H I, H II, He I, He II, He III, C II, C III, C IV, O I, O II, and O III. The reaction rates and the non-equilibrium cooling function are given in Raga et al. (2002). The calculations were performed on a four-level, binary adaptive grid with a maximum resolution along the $\mathrm{x}$, $\mathrm{y}$, and $\mathrm{z}$ axes of $\sim 50$ grid points for cloud radius (see also, Melioli, de Gouveia Dal Pino \& Raga 2004).

Klein, McKee and Colella (1994), and Poludnenko et al. (2002), for example, have performed 2-D simulations of the interaction of a strong, planar shock wave with dense inhomogeneties embedded in a more rarefied ambient medium, but their study was restricted to the adiabatic case. Since the typical timescales for radiative cooling in SBs $\left(10^{4}-10^{5} \mathrm{yr}\right)$ are much smaller than their dynamical timescales, the radiative losses are expected to have important effects on the dynamical evolution of these systems.

For a more complete study, we have also performed numerical simulations of the interactions of various SNRs inside a small SB volume $(<100 \mathrm{pc})$, These simulations have shown that the formation of very dense filaments (1000 times denser than the hot gas) and dense surfaces (100 times denser than the hot gas) is possible in times of the order of 


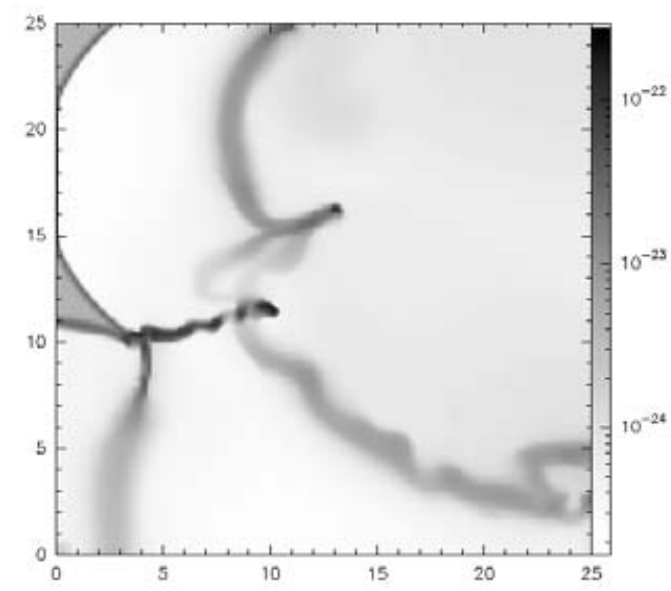

Figure 1. Grey-scale map of the density distribution showing the interaction of 5 SNRs in a box of $25 \mathrm{pc} \times 3.125 \mathrm{pc} \times 25 \mathrm{pc}$, corresponding to $256 \times 32 \times 256$ grid points at the highest grid level. The left and bottom faces that define the beginning of the computational box have reflective boundaries and the right and top faces have continuous (or outflow) boundaries. The ambient medium has $n=1 \mathrm{~cm}^{-3}$ and $\mathrm{T}=10^{4} \mathrm{~K}$. The initial energies of the SN explosions are assumed to be: $E_{0}=6.3 \times 10^{49} \mathrm{erg}, 1.6 \times 10^{50} \mathrm{erg}, 4.2 \times 10^{49} \mathrm{erg}, 3.8 \times 10^{49} \mathrm{erg}$, and $1.3 \times 10^{50}$ erg. The panel correspond to a snapshot of the evolution of the system: $t=4.5 \times 10^{4}$ yrs (from Melioli, de Gouveia Dal Pino \& Raga 2004a).

$10^{4}-10^{5}$ yrs, depending on the rate of SN explosions. For example, Figure 1 shows a case with a high SN rate, and a consequent fast formation of dense structures. Rayleigh-Taylor (R-T) and Kelvin-Helmholtz (K-H) instabilities are able to fragment these structures and produce new clouds, in a process that can continue during all the SN activity.

In general, the results have shown that, instead of an efficient gas mixing with the diffuse ISM, both SNR-clouds and SNR-SNR interactions cause the fragmentation of the shells and clouds into smaller structures. These could be associated with the dense clumps observed inside several SBs that are blown out by the galactic wind (Cecil et al. 2002). These results have also reavealed that the SNR-clouds interactions are less effective to cause large mass loss from the clouds to the diffuse ISM (and to provide a density increase in the latter) than mechanisms, such as, photoevaporation, K-H drag, and thermal evaporation. This result has important consequences on the global evolution of the SB ambient and the formation of the associated superwind (see Melioli \& de Gouveia Dal Pino 2004a, and these proceedings).

\section{References}

Gonzalez, R. F., de Gouveia Dal Pino, E. M., Raga, A., \& Velazquez, P. 2004, ApJ, 600, L59

Klein, R., McKee, C. F., \& Colella, P. 1994, ApJ, 420, 213

Masciadri, E., de Gouveia Dal Pino, E. M., Raga, A., \& Noriega-Crespo, A. 2002, ApJ, 580, 950

Melioli, C., \& de Gouveia Dal Pino, E. M. 2004a, A\&A, in press

Melioli, C., de Gouveia Dal Pino, E. M., \& Raga, A. C. 2004a, in prep.

Poludnenko, A. Y., Frank, A., \& Blackman, E. G. 2002, ApJ, 576, 832

Raga, A., Navarro-Gonzalez, R., \& Villagran-Muniz, M. 2000, RMxAA, 36, 67

Raga, A., de Gouveia Dal Pino, E. M., Noriega-Crespo, A., Minnini, P. D., \& Velázquez, P. 2002, A\&A, 392, 267 